J. Low. Temp. Phys. manuscript No.

(will be inserted by the editor)

\title{
Cooling a vibrational mode coupled to a molecular single-electron transistor
}

\author{
F. Pistolesi
}

Received: date / Accepted: date

\begin{abstract}
We consider a molecular single electron transistor coupled to a vibrational mode. For some values of the bias and gate voltage transport is possible only by absorption of one ore more phonons. The system acts then as a cooler for the mechanical mode at the condition that the electron temperature is lower than the phonon temperature. The final effective temperature of the vibrational mode depends strongly on the bias conditions and can be lower or higher of the reservoir in contact with the oscillator. We discuss the efficiency of this method, in particular we find that there is an optimal value for the electron-phonon coupling that maximizes cooling.
\end{abstract}

Keywords mesoscopic transport · NEMS · Cooling

PACS PACS 73.23.-b · PACS 73.50.Lw $\cdot 85.85 .+\mathrm{j}$

\section{Introduction}

Cooling the vibrational mode of nano-mechanical oscillator is a major challenge on the road to the observation of quantum effects in mechanical systems. Recently several proposals for cooling mechanical modes have been formulated $[1,2,3,4,5,6,7,8,8,9,10$, 11, 12, 13, 14, 15, 16] and many have been realized experimentally [17, 18, 19,20,21. A particularly efficient method is the so called sideband cooling (see for instance Refs. [5], 6]). The general idea of side-band cooling is to exploit a detuning between the frequency of a forcing field (for instance a laser source) and the resonant frequency of a cavity mode. The cavity mode is coupled to a mechanical oscillator of resonant frequency $\omega$. When the detuning is negative (the source-field frequency is lower than the cavity mode frequency) and close to $\omega$ the increase of the occupation of the cavity mode can be realized by the absorption of a phonon of the oscillator. This process removes energy from the mechanical mode and thus cools the oscillator. For positive detuning one observes the opposite phenomenon of heating due to the coherent pumping of energy in the mechanical mode.

F. Pistolesi

Laboratoire de Physique et Modélisation des Milieux Condensés,

CNRS and Universit Joseph Fourier B.P. 166, F-38042 Grenoble, France

E-mail: fabio.pistolesi@grenoble.cnrs.fr 


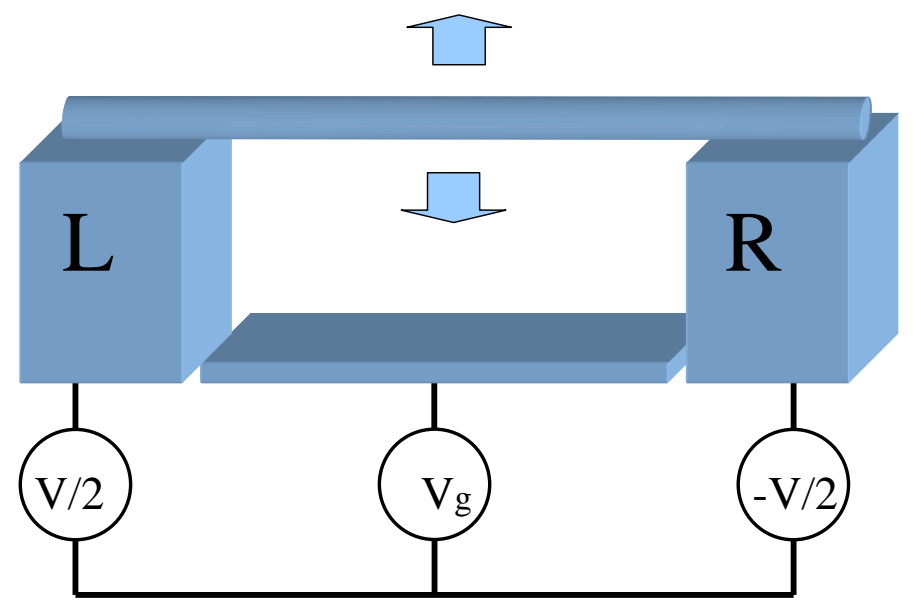

Fig. 1 An example of the system considered in the text, a suspended nanotube contacted to two leads and coupled capacitively to a gate.

In this paper we consider a similar phenomenon that can take place in a typical nano-electromechanical device: a molecular single electron transistor coupled to a mechanical oscillator (see Fig. 1). This system has been widely studied in the literature [22, $23,24,25,26,27,28,29,30,31$. In particular it has been shown that the coupling to the mechanical mode can induce a suppression of the current at low bias both in the molecular case [25 29 30] (when the size of the central island is so small that a single electronic level participates in the transport) and in the metallic classical case (when the level spacing in the island is negligible with respect to the bias voltage) 32 . The suppression of the current is known as Frank-Condon blockade or phonon-blockade, and it has a very simple classical origin: When the island is charged the force acting on it can displace the island itself leading to a different gate voltage $\left(V_{g}\right)$ seen by the device. This corresponds to a shift of the Coulomb diamonds in the $V_{g}-V$ plane ( $V$ being the bias voltage) by a small amount in the $V_{g}$ direction. The current can then flow only in the regions corresponding to the intersection of the sequential transport regions of the shifted and un-shifted diamonds. By a simple geometrical construction one finds that at low voltage current is suppressed 32. In the quantum limit $\hbar \omega \sim k_{B} T, e V$, (where $T$ is the environment temperature, $k_{B}$ is the Boltzmann constant and $e$ is the electron charge) the shift of the diamonds is discrete and each step corresponds to the absorption or the emission of phonons. The resulting Coulomb diamonds are characterized by emission 25, 29] or absorption sidebands 33. Very recently the emission sidebands predicted by these theories have been observed in suspended carbon nanotubes [34].

In this paper we discuss under which conditions it is possible to exploit the phononassisted tunneling to cool the oscillator. A necessary condition is that the electronic temperature is lower than the temperature of the environment coupled to the oscillator. This fact can be realized either because the oscillator is coupled better than the electrons to a hot environment, or by a preliminary cooling of the electrons by existing techniques of electrons cooling 35,7]. In this paper we explain how one could sequentially cool also the mechanical mode by exploiting the properties of the single electron transistor to optimize cooling. We find that it exist regions in the $V_{g}-V$ plane where the cooling effect is maximum, these are the regions just outside the conducting 
diamonds within a distance in energy of the order of $\hbar \omega$. We also find that in order to achieve a good cooling efficiency the electron-phonon coupling constant must not be too large, since then the same mechanism at the base of the phonon blockade blocks transport at low bias thus reducing also the number of absorbed phonons.

The paper is organised as follows: In Section 2 we describe in details the system and the theoretical approach in terms of a master equation for the phonon distribution. In Section 3 we give an analytical description of cooling for small bias voltage and near the border of the conducting region in the $V_{g}-V$ plane. In Section 4 we discuss the numerical solution of the master equation for several values of the parameters. Section 5 gives our conclusions.

\section{System model}

We consider large molecules (like carbon nanotubes) or quantum dots connected to two leads and in presence of a gate voltage (see Fig. 1). If the Coulomb charging energy is sufficiently high this system acts as a single electron transistor, where transport can be finely tuned by varying the gate voltage. We assume that the presence of an excess electron in the central island generates a force on a nano-oscillator, that can be a vibrational mode of the molecule itself or a nearby mobile and gated lead. We consider the regime where the oscillation frequency is larger or of the same order of the temperature, the bias and the gate voltage. This regime can be easily realized for small molecules, where the oscillating frequency can attain the $\mathrm{THz}[22,26$, in larger molecules and for metallic oscillators this can be more difficult since for instance a long carbon nanotube the oscillation frequency can be in the $\mathrm{MHz}$ range [36, 37] rendering the system classical [32. A minimal model for such a system is the Anderson-Holdstein Hamiltonian that has been considered by many authors [38, 39, 25, 27, 40:

$$
H=\left[\epsilon_{0}+\lambda \hbar \omega\left(\hat{a}+\hat{a}^{\dagger}\right)\right] \hat{d}^{\dagger} \hat{d}+\hbar \omega \hat{a}^{\dagger} \hat{a}+\sum_{k, \alpha} \epsilon_{k \alpha} \hat{c}_{k \alpha}^{\dagger} \hat{c}_{k \alpha}+\sum_{k, \alpha} t_{\alpha}\left(\hat{c}_{k \alpha}^{\dagger} \hat{d}+\hat{d}^{\dagger} \hat{c}_{k \alpha}\right)
$$

Here $\alpha$ is the lead index ( $L$ or $R$ ), $\hat{c}, \hat{c}^{\dagger}, \hat{d}$, and $\hat{d}^{\dagger}$ are the electron annihilation and creation operators for the leads $(c)$ and local orbital $(d) . \epsilon_{k \alpha}=\xi_{k}-\mu_{\alpha}$ is the dispersion relation of the single-electron bands in the lead $\alpha$ with $\mu_{\alpha}$ being the chemical potential. The index $k$ labels the single electron states in the two leads. $\epsilon_{0}$ is the energy eigenvalue of the local orbital and can be shifted by the external gate voltage (cfr. Fig. 11). The operators $\hat{a}$ and $\hat{a}^{\dagger}$ annihilate and create phonons, $\lambda$ is the electron-phonon dimensionless coupling. We consider the model for spinless electrons for simplicity. Inclusion of spin along with large onsite Coulomb repulsion leads to qualitatively similar results, as far as the Kondo temperature $T_{K} \ll \hbar \omega, k_{B} T_{e}$, with $T_{e}$ the electronic temperature. We also assume a wide band limit, so that the tunneling induced level width is simply $\Gamma_{\alpha}=\pi \nu_{\alpha} t_{\alpha}^{2}$, where $\nu_{\alpha}$ is the density of states and $t_{\alpha}$ is the tunneling amplitude in lead $\alpha$. The exact definition of the electron phonon coupling $\lambda$ depends on the explicit geometry and material parameters of the device. Values larger than unit are possible, they correspond to a displacement of the mechanical oscillator over a distance larger than the zero point motion length when an electron is added in the dot. Very recently $\lambda$ has been evaluated theoretically for the elongation modes of a suspended nanotube 34. In good agreement with the observed magnitude of the Frank-Condon sideband the authors found that $\lambda \approx 1.3$, thus exactly in the intermediate range relevant for the 
present paper. The mechanical state for $k_{B} T_{e}, \Gamma_{\alpha} \gg \hbar \omega$ has been discussed in Ref. 30, 41 for the molecular case and in Ref. [4, 43, 32, 44, for the metallic case. (The case of a single tunnel junction is discussed in Ref. 45.) The frequent hopping of electrons in and out the central island are a strong source of decoherence for the oscillator and the reduced density matrix is diagonal in the position and momentum basis of the oscillator itself. A classical approach is thus appropriate in this case. Here we consider the opposite limit of fast phonons: $\hbar \omega \gg \min \left(\Gamma_{L}, \Gamma_{R}\right)$ 25, 27, 29, 46]. The mechanical oscillator on average performs many revolutions between two electron hopping events. The oscillator reduced density matrix in first approximation is then diagonal in the base of the energy eigenvectors (phonons). The time evolution of the system can then be described by a (Pauli) master equation for the probability $P_{n p}$ that the central island is occupied by $n$ electrons (with $n=0$ or 1 in our case) and the oscillator is in the eigenstate of the free oscillator Hamiltonian with eigenvalue $n \hbar \omega$. The rates from one state to the other can be calculated with Fermi golden rule in second order in the tunneling coupling and all orders in the interaction constant $\lambda$. The standard approach is to perform a Lang-Firsov canonical transformation to eliminate the coupling term proportional to $\lambda$ from the Hamiltonian in favor of a renormalised tunneling amplitude $t_{\alpha} \rightarrow t_{\alpha} e^{-\lambda\left(\hat{a}-\hat{a}^{\dagger}\right)}$ and renormalised energy level $\epsilon_{0} \rightarrow \epsilon_{0}^{\prime}=\epsilon_{0}-\lambda^{2} \hbar \omega$. The calculation of the transition matrix elements is then straightforward. One obtains (see for instance [25, 29, 47]):

$$
\begin{aligned}
& W_{p \rightarrow p^{\prime}, \alpha}^{0 \rightarrow 1}=\frac{\Gamma_{\alpha}}{\hbar}\left|M_{p p^{\prime}}\right|^{2} f\left(\epsilon_{0}^{\prime}+\left(p^{\prime}-p\right) \hbar \omega-\mu_{\alpha}\right) \\
& W_{p \rightarrow p^{\prime}, \alpha}^{1 \rightarrow 0}=\frac{\Gamma_{\alpha}}{\hbar}\left|M_{p p^{\prime}}\right|^{2}\left[1-f\left(\epsilon_{0}^{\prime}-\left(p^{\prime}-p\right) \hbar \omega-\mu_{\alpha}\right)\right],
\end{aligned}
$$

where $W_{p \rightarrow p^{\prime}, \alpha}^{n \rightarrow n^{\prime}}$ is the transition rate from the occupation state $n$ to $n^{\prime}$ with simultaneous change of the phonon state from $p$ to $p^{\prime}$. In Eqs. (2) and (3) $f(\epsilon)=1 /\left(1+e^{\epsilon / k_{B} T_{e}}\right)$ is the Fermi distribution of the electrons in the metallic leads and $M_{p p^{\prime}}$ is the matrix element for a phonon transition. The latter is given by the overlap integral of two harmonic oscillator wavefunctions spatially displaced by $\Delta x=\lambda \sqrt{2 \hbar / m \omega}$, with $m$ the oscillator mass:

$$
\left|M_{p_{1} p_{2}}\right|=\lambda^{P-p} e^{-\lambda^{2} / 2} \sqrt{\frac{p !}{P !}} L_{p}^{P-p}\left(\lambda^{2}\right) .
$$

The absolute value of the matrix element depends only on $p=\min \left(p_{1}, p_{2}\right)$ and $P=$ $\max \left(p_{1}, p_{2}\right)$. Here $L_{p}^{P}$ are the generalized Laguerre polynomials. We neglect cotunneling events, the reader can find a detailed discussion of their contribution to the current in Ref. 47].

The mechanical oscillator is coupled to an external environment at a given temperature $T_{m}$ that we will assume in general different from the electronic temperature $T_{e}$ entering the Fermi distributions. If the environment correlation time is shorter than all the other time scales of the problems $(\Gamma / \hbar$ and $1 / \omega)$ following Ref. 29] we can model the effect of the environment with the time scale $\tau$ necessary to reach the phonon equilibrium state. The master equation takes then the form:

$$
\frac{d P_{n p}}{d t}=-P_{n p} W_{n p}+\sum_{n^{\prime} p^{\prime} \alpha} P_{n^{\prime} p^{\prime}} W_{p^{\prime} \rightarrow p, \alpha}^{n^{\prime} \rightarrow n}-\frac{1}{\tau}\left(P_{n p}-P_{p}^{e q} \sum_{p^{\prime}} P_{n p^{\prime}}\right)
$$


where $W_{n p}=\sum_{n^{\prime} p^{\prime} \alpha} W_{p \rightarrow p^{\prime}, \alpha}^{n \rightarrow n^{\prime}}$ and $P_{p}^{e q}=e^{-p \hbar \omega / k_{B} T_{m}}\left(1-e^{-\hbar \omega / k_{B} T_{m}}\right)$ is the canonical equilibrium distribution. The stationary state of the system can now be obtained by solving Eq. (5) for $d P_{n p} / d t=0$. Electronic transport can drive the oscillator out of equilibrium and in general the resulting effect can be either to increase or to decrease the average energy of the oscillator. In the next Sections we discuss the properties of the stationary solutions of Eq. (5) and the consequences for the oscillator state.

\section{Cooling by phonon absorption}

In this section we provide an analytical solution for the stationary state of the device and discuss how the bias condition on the single electron transistor can influence the thermal state of the mechanical device inducing either cooling or heating.

Since the stationary solution obtained from Eq. (5) is not in general an equilibrium distribution strictly speaking we cannot define a temperature for this state. Nevertheless one can always define the average phonon energy $E_{a v} \equiv \hbar \omega \sum_{n p} p P_{n p}$. In particular, for an equilibrium distribution one has $E_{a v}=\hbar \omega /\left(e^{\hbar \omega / k_{B} T_{m}}-1\right)$, thus an effective temperature can always be defined by inverting it:

$$
k_{B} T_{e f f} \equiv \hbar \omega / \ln \left(1+\hbar \omega / E_{a v}\right)
$$

In the following to describe the thermal state of the oscillator we will discuss either the average phonon energy or the effective temperature of the oscillator defined by Eq. (6).

Let us now discuss the form of the solution qualitatively, and in particular the contribution of the different tunneling processes. For simplicity let us assume that the electronic temperature $k_{B} T_{e} \ll \hbar \omega \sim k_{B} T_{m}$, then the Fermi functions in (2) and (3) are, within a good approximation, step functions. We define the bias voltage $e V=\mu_{L}-\mu_{R}$ and the gate voltage $e V_{g}=\left(\mu_{L}+\mu_{R}\right) / 2-\epsilon_{0}^{\prime}$ in terms of the chemical potentials of the two leads. This notation allows to recover the standard description for the single-electron transistor as a function of $V$ and $V_{g}$. For simplicity in the following we concentrate on the $V>0$ and $V_{g}>0$ case. (The results are symmetric in both variables if the tunnel junctions are symmetric.) In this case the electrons are on average transmitted from the left reservoir to the right reservoir. Sequential tunneling from the left to the right reservoir is possible only if some of the $W_{p \rightarrow p^{\prime}, L}^{0 \rightarrow 1}$ and $W_{p \rightarrow p^{\prime}, R}^{1 \rightarrow 0}$ are non-vanishing at the same time. For $T_{e}=0$ the relevant conditions are:

$$
\left\{\begin{array}{llr}
W_{p \rightarrow p^{\prime}, L}^{0 \rightarrow 0} \neq & \text { if } & e V_{g}-e V / 2<-\hbar \omega \Delta p \\
W_{p \rightarrow p^{\prime}, R}^{1 \rightarrow 0} \neq & \text { if } & -e V_{g}+e V / 2>\hbar \omega \Delta p
\end{array} .\right.
$$

In Fig. 2 we show the different regions of non-vanishing rates, their intersections divide the plane into identical rhombi. For $\Delta p=\left(p^{\prime}-p\right)=0$ Eq. (7) defines the standard Coulomb diamond conducting region in the $V_{g}-V$ plane, delimited by the two lines $V= \pm 2 V_{g}$ (dashed in Fig. 2). The conditions (7) for $\Delta p \neq 0$ divide the plane in regions where the value of the rate is constant. The variations are limited to the borders of these regions and are discontinuous.

To draw a simple picture of the mechanism of cooling we consider in the following the four relevant cases where the $V$ and $V_{g}$ are in the regions indicated in the Fig. 2 by A, B, C and D .

Region $\mathrm{A}$ is the region of maximal suppression of the current due to the electronphonon interaction [25, 29]. It is characterized also by a bistability 32,41 since in 


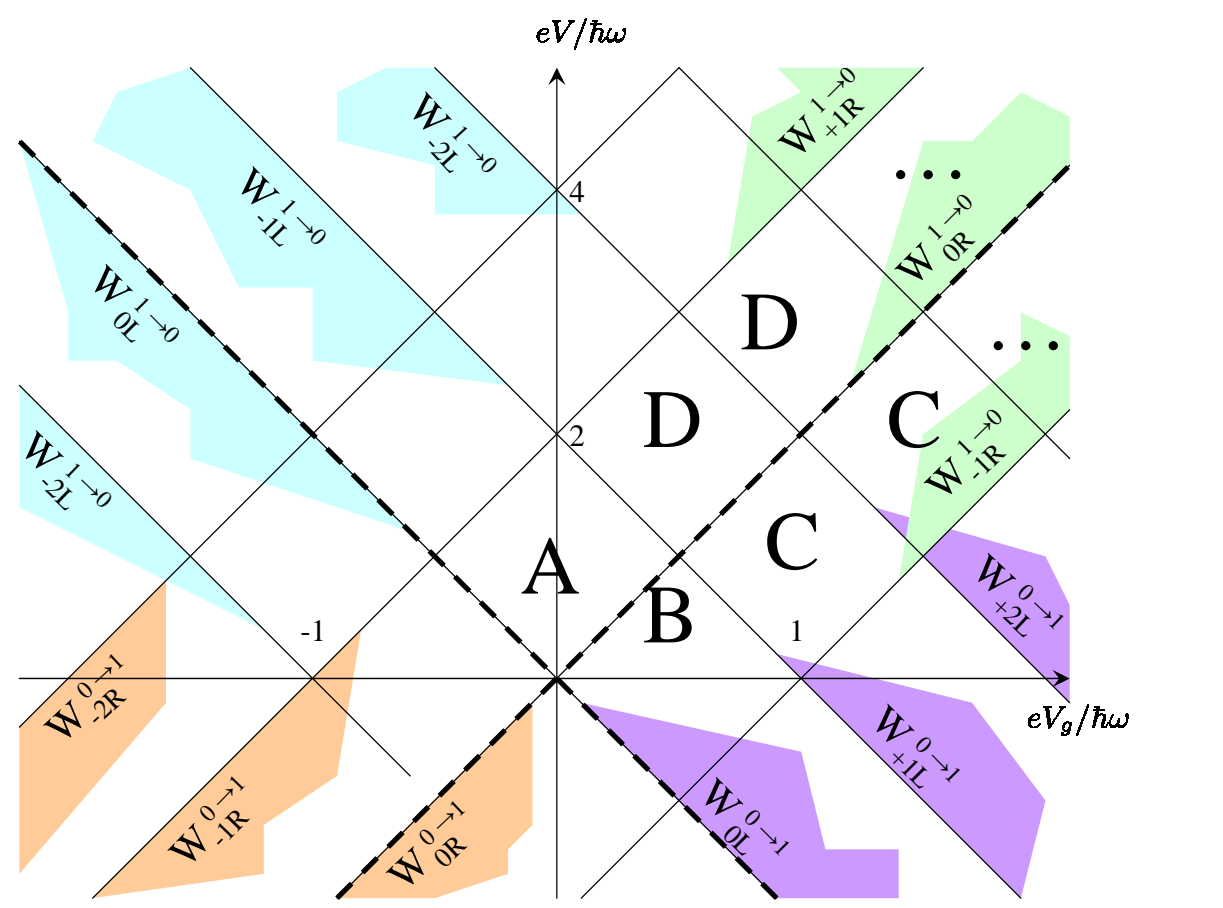

Fig. 2 (Color online) Regions of non-vanishing rates in the $V_{g}-V$ plane. The shaded regions with $W_{\Delta p \alpha}^{n \rightarrow n^{\prime}}$ indicate the regions where the rate $W_{p \rightarrow p^{\prime} \alpha}^{n \rightarrow n^{\prime}} \neq 0$ with $\Delta p=p-p^{\prime}$. The dashed line indicates the border of the sequential tunneling transport regime for the non-interacting case $\lambda=0$.

the limit of large $\lambda$ both the occupied and unoccupied states are blocked and thus stationary: the final state of the device depends then on the previous history. In order to study the stationary state we begin by assuming that only the probabilities $P_{n 0}$ and $P_{n 1}$ are significantly different from zero. This assumption has to be verified at the end of the calculation self-consistently and is equivalent to demanding that the stationary state of the oscillator is essentially the ground state with a small occupation of the first excited state. We can then solve the problem with only four states: $\underline{P}=$ $\left\{P_{00}, P_{01}, P_{10}, P_{11}\right\}$. According to the conditions (7) only few rates are non vanishing in region $\mathrm{A}$, specifically:

$$
\begin{aligned}
& W_{p \rightarrow p, L}^{0 \rightarrow 1}=W_{p \rightarrow p, R}^{1 \rightarrow 0} \equiv W_{T p} \\
& W_{1 \rightarrow 0, L}^{0 \rightarrow 1}=W_{1 \rightarrow 0, R}^{0 \rightarrow 1}=W_{1 \rightarrow 0, L}^{1 \rightarrow 0}=W_{1 \rightarrow 0, R}^{1 \rightarrow 0} \equiv W_{C}
\end{aligned}
$$

where $W_{C}=\Gamma_{0} \lambda^{2} e^{-\lambda^{2}} / \hbar$ is the relevant cooling rate $\left(\Gamma_{0} \equiv \Gamma_{L}=\Gamma_{R}\right)$. Note a crucial point, all the rates increasing the number of phonons (and thus leading to heating) are vanishing. Only the elastic transport rates (8), or the cooling rates (9) are present, thus one can easily anticipate that the system will act as a cooler in this regime. The master equation can be written in matrix form as follows $d \underline{P} / d t=M \underline{P}$ where the matrix $M$ 
reads

$$
\left(\begin{array}{cccc}
-W_{T 0}-P_{1}^{e q} / \tau & P_{0}^{e q} / \tau & W_{T 0} & 2 W_{C} \\
P_{1}^{e q} / \tau & -W_{T 1}-2 W_{C}-P_{0}^{e q} / \tau & 0 & W_{T 1} \\
W_{T 0} & 2 W_{C} & -W_{T 0}-P_{1}^{e q} / \tau & P_{0}^{e q} / \tau \\
0 & W_{T 1} & P_{1}^{e q} / \tau & -W_{T 1}-2 W_{C}-P_{0}^{e q} / \tau
\end{array}\right)
$$

and we have introduced the terms due to the coupling of the oscillator to the external environment. The stationary solution is given by the eigenvector of vanishing eigenvalue of the matrix $M$ that reads

$$
\underline{P}=\left\{P_{0}^{e q}+2 \tau W_{C}, P_{1}^{e q}, P_{0}^{e q}+2 \tau W_{C}, P_{1}^{e q}\right\} .
$$

It gives for the occupation of the excited state $P_{q} \equiv P_{0 q}+P_{1 q}$ :

$$
P_{1}=\frac{P_{1}^{e q}}{1+2 W_{C} \tau}
$$

This expression has two simple limits: For $\tau \rightarrow 0$ the environment of the oscillator dominates and the system remains in thermal equilibrium with his own bath. For $\tau \rightarrow \infty$, the system reaches its ground state, since $P_{1} \rightarrow 0$. In general the relevant parameter to determine which of the two cases is realized is the product $\tau W_{C}$. It is maximum for $\lambda=1$ that thus constitutes in this region the optimal value of the electron-phonon coupling for cooling efficiency. Specifically, for this value of $\lambda, P_{1}$ reads $P_{1}^{e q} /\left(1+2 \Gamma_{0} \tau / e \hbar\right)$. The condition for efficient cooling is then simply $\Gamma_{0} \tau / \hbar \gg 1$.

To understand better the behavior of the device let us now consider the three other regions next to the phonon blockade area: regions $\mathrm{B}, \mathrm{C}$ and $\mathrm{D}$, as shown in Fig. 2 . In region $\mathrm{B}$ the situation is very similar to region $\mathrm{A}$, the non vanishing rates are

$$
\begin{aligned}
& W_{p \rightarrow p, L}^{0 \rightarrow 1}=W_{p \rightarrow p, R}^{0 \rightarrow 1} \equiv W_{T p} \\
& W_{1 \rightarrow 0, L}^{0 \rightarrow 1}=W_{1 \rightarrow 0, R}^{0 \rightarrow 1}=W_{1 \rightarrow 0, L}^{1 \rightarrow 0}=W_{1 \rightarrow 0, R}^{1 \rightarrow 0} \equiv W_{C} .
\end{aligned}
$$

Again we have no heating terms, the only difference is in the elastic transport terms (13). We find that the stationary solution for $P_{1}$ has the form (11) as in the the region A. The final effective temperature of region $\mathrm{B}$ thus coincides with that of region $\mathrm{A}$.

Let us now consider region $\mathrm{C}$, this is an important case since one can show that actually all the blocks delimited by the two lines $\mathrm{eV}=2 \mathrm{e} V_{g}$ and $\mathrm{eV}=2 \mathrm{e} V_{g}+2 \hbar \omega$ for $e V_{g}>-2 e V+\hbar \omega$ (indicated as $C$ in Fig. 2) have exactly the same behavior. The list of non-vanishing rates reads:

$$
\begin{aligned}
& W_{p \rightarrow p, L}^{0 \rightarrow 1}=W_{p \rightarrow p, R}^{0 \rightarrow 1} \equiv W_{T p} \\
& W_{1 \rightarrow 0, L}^{0 \rightarrow 1}=W_{1 \rightarrow 0, R}^{0 \rightarrow 1}=W_{1 \rightarrow 0, R}^{1 \rightarrow 0} \equiv W_{C} \\
& W_{0 \rightarrow 1, L}^{0 \rightarrow 1} \equiv W_{H}=W_{C} .
\end{aligned}
$$

The situation is different from case $\mathrm{A}$ and $\mathrm{B}$, since now one phonon-emission rate is non vanishing [Eq. (17)]. Still there are three phonon absorption terms (16) and we can expect that the final result will be not far from the ground state. Also in this case it is possible to find the stationary solution analytically, but its form is rather cumbersome, we prefer to present here only the result for large $\tau$ :

$$
P_{1} / P_{1}^{e q}=\frac{W_{C}+2 W_{T 0}}{2 \tau W_{C} W_{T 0}}=\left(\hbar / \tau \Gamma_{0}\right) e^{\lambda^{2}}\left(2+\lambda^{2}\right) / 2 \lambda^{2} .
$$


We thus find again that for large $\tau$ the single electron transistor behaves as a good cooler for the oscillator. It is important to notice that this result holds for large $e V$, since all the blocks along the threshold of current behave in the same way. This holds for any value of $\lambda$, but the optimal range for cooling is $\lambda \approx 1$. We can now check the self-consistency of the solution. The runcation of the space to the first excited state is justified by the fact that $P_{1} \sim \Gamma_{0} / \tau$ vanishes for $\tau \Gamma_{0} \rightarrow \infty$.

Let us finally check what happens when the system is biased in region $\mathrm{D}$, where the sequential transport channel is open. Only the elastic rates differ from the set of non-vanishing rates of region C [Eq.: (15) is substituted by the following Equation]

$$
W_{p \rightarrow p, L}^{0 \rightarrow 1}=W_{p \rightarrow p, R}^{1 \rightarrow 0} \equiv W_{T p} .
$$

This difference is very important, since now it is possible to let an electron out of the central island by elastic tunneling $\left(W_{q \rightarrow q, R}^{1 \rightarrow 0}\right)$ and let an other electron in by emitting a phonon $\left(W_{0 \rightarrow 1, L}^{0 \rightarrow 1}\right)$. This process heats up the device and is in competition with the cooling processes. In region $\mathrm{C}$ the only way for an electron to exit the central island is by absorption of one phonon by the oscillator $\left(W_{1 \rightarrow 0, R}^{1 \rightarrow 0}\right)$, thus also if an electron could enter the device by emitting one phonon $W_{0 \rightarrow 1, L}^{0 \rightarrow 1}$ the net balance of the two processes is always of no energy exchanged. Since all the other processes imply cooling of the oscillator the stationary solution is a cold state. In region $D$ this is no more true, and we can verify it by finding the stationary state for $\tau=\infty$. We find that $P_{1}$ does not vanish in this limit:

$$
P_{1}=\frac{W_{T 0}\left(W_{C}+W_{T 1}\right)}{4 W_{T 0} W_{T 1}+W_{C}\left(3 W_{T 0}+W_{T 1}\right)} .
$$

Note also that this result for $P_{1}$ is not the accurate stationary solution, since the fact that it does not vanish for large $\tau$ invalidate the truncation of the space to the the first two phonon states. To find the true stationary solution we have to include more states, this can be easily done numerically. The result will be discussed in the next Section, but it is clear that the system cannot be cooled at very low temperature in region $\mathrm{D}$. It is also clear that going even more deeply inside the sequential tunneling regime the situation will not improve, and finally one would find that electronic transport heats up the oscillator.

\section{Numerical results}

In order to confirm the analytical results and to investigate their limitations in this Section we discuss the numerical solution of the full master equation (5). This can be done by truncating the dimension of the space to $2 p_{M}$ states such that the $e V, e V_{g}, k_{B} T_{m}$, $k_{B} T_{e} \ll p_{M} \hbar \omega$. In practice in the following $p_{M}=30$ will be sufficient for our purposes.

We begin by considering an ideal case of very cold electrons $k_{B} T_{e} \ll \hbar \omega$, and long relaxation rate of the phonons $\tau \Gamma_{0} / \hbar=100$, with $\Gamma_{0} / \hbar \omega=0.1$ and $\lambda=1$. The resulting effective temperature of the oscillator is shown as a function of the bias and gate voltage in Fig. 3 The plot shows how important are the bias condition, since the effective temperature dependence clearly allows to recognize the different regions we discussed in Section 3 since the temperature changes abruptly at their borders. The results confirm the analysis of the previous Section, regions A, B, and the stripe of $\mathrm{C}$ regions correspond to a stationary solution with very low effective temperature 


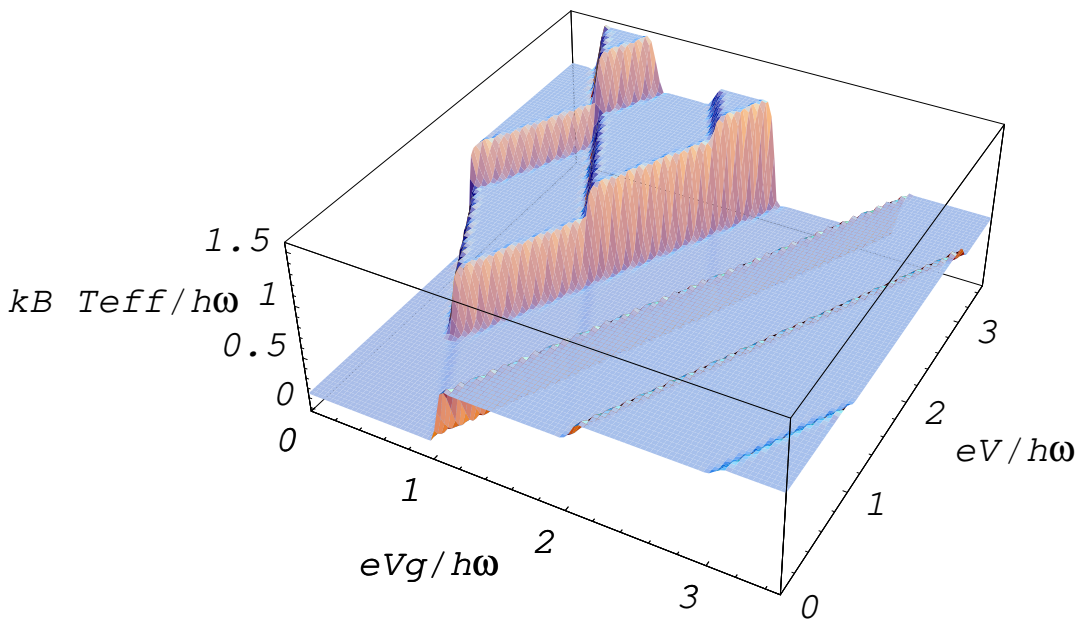

Fig. 3 (Color online) Effective temperature of the oscillator in the $e V_{g}-e V$ plane. In this calculation $T_{e} / \hbar \omega=0.01, T_{m}=\hbar \omega, \tau / \Gamma_{0}=100, \lambda=1$, and $\Gamma_{0} / \omega=0.1$. We assume symmetric junctions. In this figure it is easy to recognize the different regions shown in Fig. 2

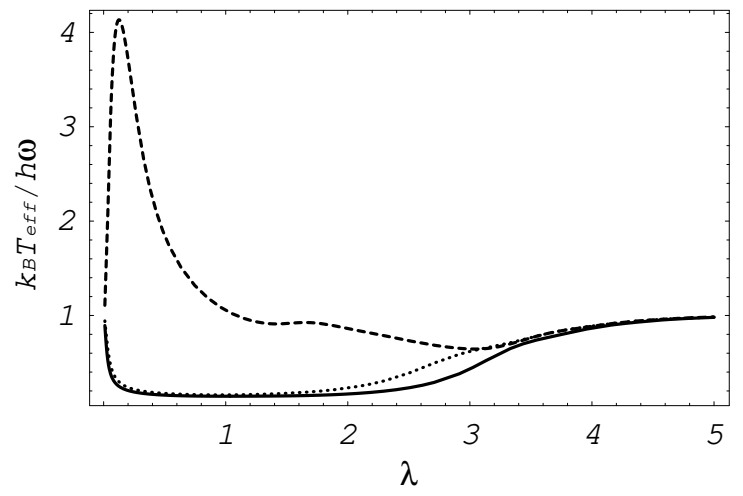

Fig. 4 Effective temperature as a function of the coupling constant $\lambda$ for the same parameters of Fig. 3 for different bias points. Specifically the full, dotted, and dashed lines correspond to the bias points $\left(e V_{g}, e V\right) / \hbar \omega=(0,0.5),(0,2.5)$, and $(1,0.5)$, respectively.

(cfr. Fig. 3). The effective temperature is much smaller than the mechanical reservoir temperature. In particular the lowest temperature is obtained for any of the $C$ regions, regardless of the value of the bias voltage. We also can see that the temperature is higher in the D rhombi for the reasons explained in Section 3

In Fig. 4 we plot the coupling dependence of the effective temperature for the same parameters of the Fig. 3 at different bias points as indicated in the caption. The full 


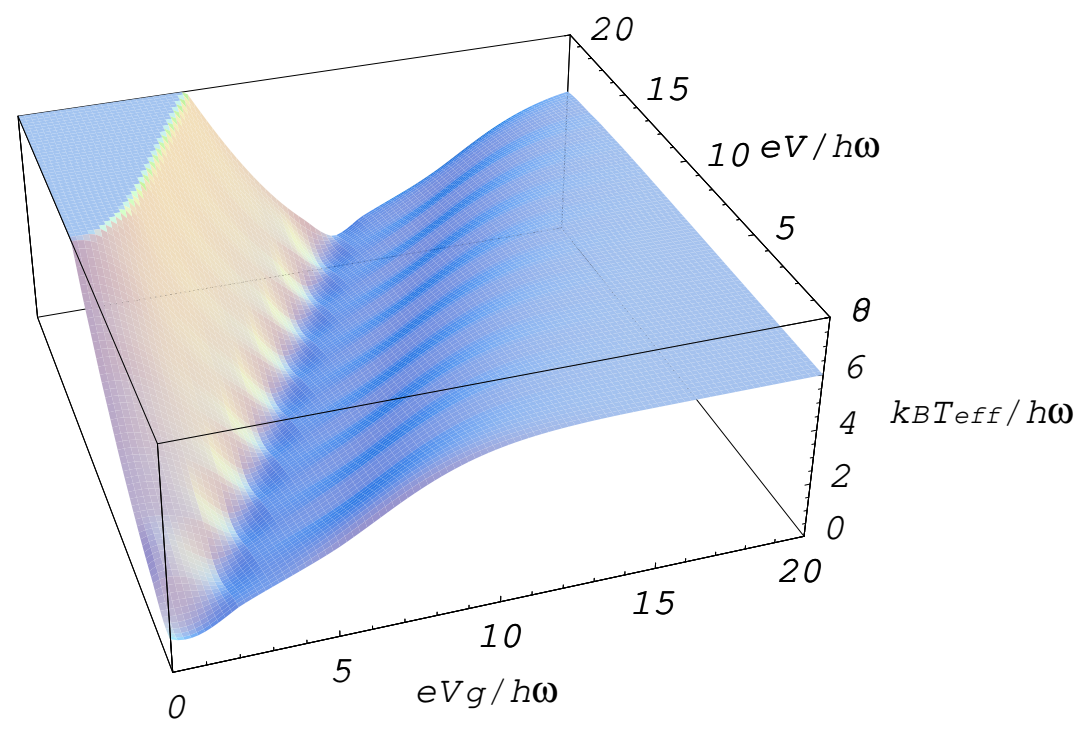

Fig. 5 (Color online) Effective temperature for $T_{e} / \hbar \omega=0.1, T_{m} / \hbar \omega=5, \tau / \Gamma_{0}=100, \lambda=1$, and $\Gamma_{0} / \omega=0.1$. Compared to Fig. 3 we plot here a much larger region of the plane, since the temperature is higher.

and dotted line correspond to the case A, and C, respectively. We see that the optimal cooling is attained for $\lambda=1$, as found in the analytic calculation. The dashed line shows the behavior in the middle of the Coulomb diamond, and for bias voltage larger that $2 \hbar \omega$, that constitutes the inelastic threshold in this case. We see that an increase of the coupling leads to a strong heating of the device, which is expected since the electrons have enough energy to release one phonon in the oscillator every time they traverse the device. For very large coupling the effect is suppressed by the reduction of all the rates due to the exponential vanishing of the overlap of the harmonic oscillator wavefunctions.

Finally let us consider the case of $T_{m}=5 \hbar \omega$ and still cold electrons $T_{e}=0.1 \hbar \omega$. The effective temperature for this case (the other parameters are the same of Fig. 3) is shown in Fig. 5 . The main feature is clearly the presence of a deep trench at the border of the conducting diamond. Again this is in agrement with the analytical results, that shows that $T_{\text {eff }}$ should not depend on $\mathrm{eV}$ along that line. We also see that in the conducting region for large enough voltages the current through the device heats up the oscillator, as is usually the case. On the other side, too far from the conducting region the temperature is not modified since transport is completely suppressed.

As a final check we also verified that when the electronic temperature $T_{e}$ coincides with $T_{m}$, cooling is not possible. There is always a dependence of the effective temperature of the stationary state on the bias condition, and in some case we find a very small reduction of the temperature of the oscillator, but clearly in this regime the device could not be used as a cooler for the oscillator. 


\section{Conclusions}

We have shown that a molecular (single electronic level) single-electron transistor coupled to an oscillator can act as a cooler for the oscillator if the electronic temperature is lower than the temperature of the environment to which the oscillator is coupled. The heat transport mechanism is not trivial since it involves the phonon absorption and emission, that can be finely tuned by the bias condition of the single electron transistor. We find that optimal cooling is achieved by biasing the device near the border of the sequential tunneling conducting region of the $V_{g}$ - $V$ plane. By using for instance normal-metal-superconductor tunnel junctions electronic cooler, it is possible to generate cold sources of electrons and then to exploit them to cool nano-electromechanical oscillators.

Acknowledgements I acknowledge a stimulating discussion on this problem with A. Armour and M. Houzet. This work has been supported by the French Agence Nationale de la Recherche under contract ANR-06-JCJC-036 NEMESIS. I thank A. Buzdin and his group for hospitality at the Centre de Physique Moleculaire Optique et Hertzienne of Bordeaux (France) where part of this work has been completed.

\section{References}

1. I. Martin, A. Shnirman, L. Tian, P. Zoller, Phys. Rev. B 69(12), 125339 (2004)

2. I. Wilson-Rae, P. Zoller, A. Imamoglu, Phys. Rev. Lett. 92(7), 075507 (2004)

3. A.A. Clerk, S. Bennet, New J. Phys. 7, 238 (2005)

4. M.P. Blencowe, J. Imbers, A.D. Armour, New J. Phys. 7, 236 (2005)

5. I. Wilson-Rae, N. Nooshi, W. Zwerger, T.J. Kippenberg, Phys. Rev. Lett. 99(9), 093901 (2007)

6. F. Marquardt, J.P. Chen, A.A. Clerk, S.M. Girvin, Phys. Rev. Lett. 99(9), 093902 (2007)

7. S. Rajauria, P.S. Luo, T. Fournier, F.W.J. Hekking, H. Courtois, B. Pannetier, Phys. Rev. Lett. 99(4), 047004 (2007)

8. D.A. Rodrigues, J. Imbers, A.D. Armour, Phys. Rev. Lett. 98(6), 067204 (2007)

9. C. Genes, D. Vitali, P. Tombesi, S. Gigan, M. Aspelmeyer, Phys. Rev. A 77(3), 033804 (2008)

10. A. Dantan, C. Genes, D. Vitali, M. Pinard, Phys. Rev. A 77(1), 011804 (2008)

11. C. Metzger, I. Favero, A. Ortlieb, K. Karrai, Phys. Rev. B 78(3), 035309 (2008)

12. F.W.J. Hekking, A.O. Niskanen, J.P. Pekola, Phys. Rev. B 77(3), 033401 (2008)

13. J. Hauss, A. Fedorov, C. Hutter, A. Shnirman, G. Schön, Phys. Rev. Lett. 100(3), 037003 (2008)

14. Y.D. Wang, K. Semba, H. Yamaguchi, New J. Phys. 10(4), 043015 (2008)

15. V. Koerting, T.L. Schmidt, C.B. Doiron, B. Trauzettel, C. Bruder, arXiv:0810.5718 (2008)

16. S. Zippilli, G. Morigi, A. Bachtold, arXiv:0811.2942 (2008)

17. A. Naik, O. Buu, M. LaHaye, A. Armour, A. Clerk, M. Blencowe, K. Schwab, Nature 443 $193(2006)$

18. A. Schliesser, P. Del'Haye, N. Nooshi, K.J. Vahala, T.J. Kippenberg, Phys. Rev. Lett. 97(24), $243905(2006)$

19. Arcizet, P.F. Cohadon, T. Briant, M. Pinard, A. Heidmann, Nature 444, 71 (2006)

20. A. Vinante, M. Bignotto, M. Bonaldi, M. Cerdonio, L. Conti, P. Falferi, N. Liguori, S. Longo, R. Mezzena, A. Ortolan, G.A. Prodi, F. Salemi, L. Taffarello, G. Vedovato, S. Vitale, J.P. Zendri, Phys. Rev. Lett. 101(3), 033601 (2008)

21. J.D. Teufel, C.A. Regal, K.W. Lehnert, New J. Phys. 10, 095002 (2008)

22. H. Park, J. Park, A. Lim, E. Anderson, A. Alivisatos, P. McEuen, Nature 407, 57 (2000)

23. D. Boese, H. Schoeller, Europhys. Lett. 54, 668 (2001)

24. A. Nitzan, M.A. Ratner, Science 300, 1384 (2003)

25. S. Braig, K. Flensberg, Phys. Rev. B 68, 205324 (2003)

26. B.J. LeRoy, S.G. Lemay, J. Kong, C. Dekker, Appl. Phys. Lett. 84, 4280 (2004)

27. A. Mitra, I. Aleiner, A.J. Millis, Phys. Rev. B 69, 245302 (2004) 
28. Ya. M. Blanter, O. Usmani, and Y. V. Nazarov, Phys. Rev. Lett. 93, 136802 (2004). Ibid 94, 049904(E) (2005).

29. J. Koch, F. von Oppen, Phys. Rev. Lett. 94, 206804 (2005)

30. D. Mozyrsky, M.B. Hastings, I. Martin, Phys. Rev. B 73(3), 035104 (2006)

31. A. Zazunov, D. Feinberg, T. Martin, Phys. Rev. B 73(11), 115405 (2006)

32. F. Pistolesi, S. Labarthe, Phys. Rev. B 76, 165317 (2007)

33. M.C. Lüffe, J. Koch, F. von Oppen, Phys. Rev. B 77(12), 125306 (2008)

34. R. Leturcq, C. Stampfer, K. Inderbitzin, L. Durrer, C.Hierold, E. Mariani, M.G. Schultz, F. von Oppen, K. Ensslin, unpublished (2008)

35. M.M. Leivo, J.P. Pekola, D.V. Averin, App. Phys. Lett. 68(14), 1996 (1996)

36. V. Sazonova, Y. Yaish, H. Ustunel, D. Roundy, A. Arias, P.M. McEuen, Nature 431, 284 (2004)

37. S. Sapmaz, P. Jarillo-Herrero, Y.M. Blanter, C. Dekker, H.S.J. van der Zant, Phys. Rev. Lett. 96, 026801 (2006)

38. L.I. Glazman, R.I. Shekhter, Soviet Physics - JETP 67(1), 163 (1988)

39. N.S. Wingreen, K.W. Jacobsen, J.W. Wilkins, Phys. Rev. B 40(17), 11834 (1989)

40. A. Zazunov, T. Martin, Phys. Rev. B 76(3), 033417 (2007)

41. F. Pistolesi, Y.M. Blanter, I. Martin, Phys. Rev. B 78(8), 085127 (2008)

42. A.D. Armour, M.P. Blencowe, Y. Zhang, Phys. Rev. B 69, 125313 (2004)

43. C.B. Doiron, W. Belzig, C. Bruder, Phys. Rev. B 74, 205336 (2006)

44. O. Usmani, Y.M. Blanter, Y.V. Nazarov, Phys. Rev. B 75, 195312 (2007)

45. N. Pauget, F. Pistolesi, M. Houzet, Phys. Rev. B 77(23), 235318 (2008)

46. D.A. Ryndyk, G. Cuniberti, Phys. Rev. B 76(15), 155430 (2007)

47. J. Koch, F. von Oppen, A.V. Andreev, Phys. Rev. B 74(20), 205438 (2006) 